\title{
INFINITE LINEAR DIFFERENCE EQUATIONS WITH ARBITRARY REAL SPANS AND FIRST DEGREE COEFFICIENTS
}

\author{
BY \\ R. H. CAMERON AND W. T. MARTIN
}

1. Introduction. Finite difference equations with arbitrary real spans have been investigated by various mathematicians $\left({ }^{1}\right)$. In this paper we shall consider infinite difference equations with arbitrary real spans whose coefficients are either constants or linear functions of the independent variable.

We shall state at the outset three theorems which give some indication of the scope of our work. Theorems 1, 2 and 3 are special cases of Theorems 1a, $2 \mathrm{a}$ and $3 \mathrm{a}$ which will be proved in the body of the paper. We state these special cases explicitly because they are simpler and can be expressed in the usual terminology of difference equations. The three theorems are not independent since 1 and 2 together imply 3 , and 2 is a special case of 3 . Nevertheless, it seems worthwhile to quote 1 and 2 separately because of their greater simplicity and because they form the natural introduction to Theorem 3 . They deal, respectively, with the case of constant coefficients, the case of all coefficients constant but one (which is linear), and the case of all linear coefficients.

THEOREM 1. Let

$$
\sum_{n=1}^{\infty} a_{n} f\left(z-\lambda_{n}\right)=g(z)
$$

be an infinite difference equation in which the $\lambda_{n}$ are arbitrary real numbers and the $a_{n}$ are complex numbers such that

$$
\sum_{n=1}^{\infty} a_{n} e^{\lambda_{n} w}
$$

converges absolutely to a non-vanishing function $A(w)$ in some strip $\alpha<\operatorname{Re} w$ $<\beta$. Furthermore let $g(z)$ be analytic in some strip $a<\operatorname{Im} z<b$. If for some $\epsilon>0$ inequalities

Presented to the Society, October 31, 1942; received by the editors June 24, 1942.

(1) A treatment of a very general nature has recently been given by $\mathrm{T}$. Kitagawa, On the theory of linear translatable functional equations and Cauchy's series, Jap. J. Math. vol. 13 (1937) pp. 233-332. Kitagawa gives other references to the literature. The methods used in the treatment of the first theorem of the present paper are more closely related to those of $\mathrm{S}$. Bochner, Allgemeine lineare Differenzengleichungen mit asymptotisch konstanten Koeffizienten, Math. Zeit. vol. 33 (1931) pp. 426-450, and W. T. Martin, Linear difference equations with arbitrary real spans, Acta Math. vol. 69 (1937) pp. 57-98. The other theorems use somewhat different methods. 


$$
\left.\begin{array}{l}
|g(x+i y)| e^{\beta^{\prime} x}<K_{\alpha^{\prime}, \beta^{\prime}, a^{\prime}, b^{\prime}} \text { for } \quad x<0 \\
|g(x+i y)| e^{\alpha^{\prime} x}<K_{\alpha^{\prime}, \beta^{\prime}, a^{\prime}, b^{\prime}} \text { for } x>0
\end{array}\right\} a^{\prime}<y<b^{\prime}
$$

hold for every $\alpha^{\prime}, \beta^{\prime}, a^{\prime}, b^{\prime}$ in

$$
\alpha<\alpha^{\prime}<\alpha+\epsilon, \quad \beta-\epsilon<\beta^{\prime}<\beta, \quad a<a^{\prime}<b^{\prime}<b,
$$

then it follows that (1.1) has one and only one solution $f(z)$ of the same character, that is, analytic in $a<\operatorname{Im} z<b$ and satisfying

$$
\left.\begin{array}{l}
|f(x+i y)| e^{\beta^{\prime} x}<K_{\alpha^{\prime}, \beta^{\prime}, a^{\prime}, b^{\prime}}^{\prime} \text { for } x<0 \\
|f(x+i y)| e^{\alpha^{\prime} x}<K_{\alpha^{\prime}, \beta^{\prime}, a^{\prime}, b^{\prime}}^{\prime} \text { for } x>0
\end{array}\right\} a^{\prime}<y<b^{\prime}
$$

for every $\alpha^{\prime}, \beta^{\prime}, a^{\prime}, b^{\prime}$ in (1.4). Moreover $f(z)$ is given explicitly by the formula

where

$$
f(z)=\sum_{n=1}^{\infty} a_{n}^{\prime} g\left(z-\lambda_{n}^{\prime}\right), \quad a<\operatorname{Im} z<b,
$$

$$
\frac{1}{A(w)}=\sum_{n=1}^{\infty} a_{n}^{\prime} e^{\lambda_{n}^{\prime} w}, \quad \quad \alpha<\operatorname{Re} w<\beta\left({ }^{2}\right) .
$$

THEOREM 2. Let

$$
z f(z)=\sum_{n=1}^{\infty} b_{n} f\left(z-\lambda_{n}\right)+g(z)
$$

be a difference equation in which the $\lambda_{n}$ are arbitrary real numbers and the $b_{n}$ are complex numbers such that

$$
\sum_{n=1}^{\infty} b_{n} e^{\lambda_{n} w}
$$

converges absolutely to a function $B(w)$ in some strip $\alpha<\operatorname{Re} w<\beta$ of the auxiliary w-plane. Let $y_{0}$ be the imaginary part of the constant term of (1.7) (zero if it has none). Then if $g(z)$ is analytic and satisfies (1.3) where the strip $a<\operatorname{Im} z<b$ does not contain the line $\operatorname{Im} z=y_{0}$, it follows that (1.6) has a unique solution $f(z)$ of the same character, that is, analytic in $a<\operatorname{Im} z<b$ and satisfying (1.5).

Theorem 3. Let

$$
\sum_{n=1}^{\infty}\left(a_{n} z+b_{n}\right) f\left(z-\lambda_{n}\right)=g(z)
$$

(2) Throughout this paper two complex planes will enter into consideration, a $z$-plane $(z=x+i y)$ in which the independent variable in our functional equation ranges, and an auxiliary $w$-plane $(w=u+i v)$ in which we consider an associated function which governs the growth of the solutions in the $z$-plane. 
be a difference equation involving arbitrary real spans and complex linear coefficients such that

$$
\sum_{n=1}^{\infty} a_{n} e^{\lambda_{n} w} \quad \text { and } \quad \sum_{n=1}^{\infty}\left(b_{n}+a_{n} \lambda_{n}\right) e^{\lambda_{n} w}
$$

converge absolutely to functions $A(w)$ and $\tilde{B}(w)$ in the strip $\alpha<\operatorname{Re} w<\beta$, the function $A(w)$ being non-vanishing in the strip. Then

$$
y_{0}=-\operatorname{Im}\left\{\lim _{T \rightarrow \infty} \frac{1}{T i} \int_{\gamma}^{\gamma+T_{i}} \frac{\tilde{B}(w)}{A(w)} d w\right\}
$$

will exist and be independent of $\gamma(\alpha<\gamma<\beta)$; and if $g(z)$ is analytic and satisfies (1.3) where the strip $a<\operatorname{Im} z<b$ does not contain the line $\operatorname{Im} z=y_{0}$, it follows that (1.8) has a unique solution $f(z)$ of the same character, that is, analytic in and satisfying (1.5).

The difference equations which we have just stated can all be expressed in terms of Stieltjes integrals. The difference equations are therefore special cases of certain Stieltjes integral equations, which are dealt with in Theorems $1 \mathrm{a}, 2 \mathrm{a}$ and $3 \mathrm{a}$. These theorems are proved by means of the theory of Fourier-Stieltjes transforms. Theorem 1a which depends on Pitt's lemma $\left.{ }^{3}\right)$ is in a certain sense not a true Stieltjes integral theorem since the Stieltjes integral involved is restricted to have no singular part, and the equation is thus really only an infinite integro-difference equation. The same is true of Theorem 3a insofar as it depends upon Theorem 1a. No such restriction is required in Theorem 2a, and it is therefore strictly a Stieltjes integral equation theorem.

If we form a right-continuous step function $a(\lambda)$ with $a(0)=0$ and with jumps of $a_{n}$ at points $\lambda_{n}$,

$$
a(\lambda)=\left\{\begin{array}{cl}
\sum_{0<\lambda_{n}<\lambda} a_{n}, & \lambda \geqq 0, \\
-\sum_{\lambda<\lambda_{n}<0} a_{n}, & \lambda<0,
\end{array}\right.
$$

then the condition that the series (1.2) be absolutely convergent in $\alpha<\operatorname{Re} w$ $<\beta$ can be expressed in an alternative but equivalent form.

Lemma 1.1. A necessary and sufficient condition that the series (1.1) be absolutely convergent in $\alpha<\operatorname{Re} w<\beta$ is that

(3) H. R. Pitt, Mercerian theorems, Proc. Cambridge Philos. Soc. vol. 34 (1938) pp. 510-520. See Lemma, p. 513. See also I. Gelfand, Über absolut konvergente trigonometrische Reihen und Integrale, Rec. Math. (Mat. Sbornik) N.S. vol. 9 (1941) pp. 51-66. Equation (3.4) of Theorem 1a has been solved by Pitt on the real line in the above paper and in General Mercerian theorems (II), Proc. London Math. Soc. vol. 47 (1942) pp. 248-267. 


$$
\int_{-\infty}^{0} e^{\alpha^{\prime} \lambda}|d a(\lambda)|+\int_{0}^{\infty} e^{\beta^{\prime} \lambda}|d a(\lambda)|<\infty
$$

for every $\alpha^{\prime}, \beta^{\prime}$ in $\alpha<\alpha^{\prime}<\beta^{\prime}<\beta$.

Also the sum-function $A(w)$ of (1.2) is given by $A(w)=\int_{-\infty}^{\infty} e^{\lambda w} d a(\lambda)$, $\alpha<\operatorname{Re} w<\beta$.

The growth condition (1.3) can also be expressed in an alternative and equivalent form, as we shall show.

LEMmA 1.2. Let $g(z)$ be analytic in a strip $a<y<b,-\infty<x<\infty$, and let $\alpha, \beta, \epsilon$ be given real numbers with $\epsilon>0$. Then a necessary and sufficient condition that (1.3) shall hold for every $\alpha^{\prime}, \beta^{\prime}, a^{\prime}, b^{\prime}$ in (1.4) is that

$$
\sup _{a^{\prime}<\nu<b^{\prime}}\left[\int_{-\infty}^{0} e^{\beta^{\prime} x}|g(x+i y)| d x+\int_{0}^{\infty} e^{\alpha^{\prime} x}|g(x+i y)| d x\right]<\infty
$$

hold for every $\alpha^{\prime}, \beta^{\prime}, a^{\prime}, b^{\prime}$ in (1.4).

Lemma 1.2 is an immediate consequence of the following property of analytic functions, which is based upon the Phragmén-Lindelöf principle:

Let $h(z)$ be analytic in the half-strip

$$
c \leqq \operatorname{Im} z \leqq d, \quad 0 \leqq \operatorname{Re} z<\infty,
$$

and let

$$
\int_{0}^{\infty}|h(x+i y)| d x \leqq M,
$$$$
c \leqq y \leqq d
$$

Then for every strip $\left(c^{\prime}, d^{\prime}\right)$ interior to $(c, d)$ we have

$$
|h(x+i y)|<M_{c^{\prime}, d^{\prime}}, \quad \quad c^{\prime}<y<d^{\prime}, 0 \leqq x .
$$

Proof. Let $z$ be any point in (1.13) and define

$$
H(z)=\int_{i c}^{z} h(t) d t
$$

The function $H(z)$ so defined is analytic in (1.13) and is independent of the path as long as the path lies in (1.13). On taking the path to be the sum of two straight-line segments $(i c, i \operatorname{Im} z)$ and $(i \operatorname{Im} z, z)$ we find

$$
|H(z)| \leqq \int_{c}^{d}|h(i y)| d y+\int_{0}^{x}|h(u+i y)| d u \leqq M_{1}+M .
$$

Thus $H(z)$ is bounded in (1.13). Moreover by (1.14) we see that $H(z)$ approaches a limit as $z$ approaches infinity along any one horizontal half-line in $c \leqq \operatorname{Im} z \leqq d$. Thus by a well known form of the Phragmén-Lindelöf theorem $H(z)$ approaches one limit value uniformly in the strip (1.13). 
Next if $c<c^{\prime}<d^{\prime}<d$ and $\delta$ is a sufficiently small positive number, then for $c^{\prime}<y<d^{\prime}$ and $\delta<x$,

$$
\begin{aligned}
h(x+i y) & =\frac{1}{2 \pi i} \int_{|t-z|=\delta} \frac{H(t)}{(t-z)^{2}} d t \\
& =\frac{1}{2 \pi} \int_{0}^{2 \pi} \frac{H\left(z+\delta e^{i \phi}\right)}{\delta e^{i \phi}} d \phi \rightarrow \text { (const.) } \frac{1}{\delta} \int_{0}^{2 \pi} e^{-i \phi} d \phi=0,
\end{aligned}
$$

as $x \rightarrow \infty$, the convergence being uniform in $c^{\prime}<y<d^{\prime}$. Thus (1.15) holds.

2. Norms and their properties. Before giving the necessary lemmas and definitions for our work on integral equations we will make a few remarks which indicate the motivation for the remainder of the section. In order to include the previously stated theorems on difference equations under our theorems on integral equations, as well as to have more generality in the integral equations themselves, we must deal with functions $p(\lambda)$ of bounded variation on every finite interval but not necessarily on the whole infinite interval. Thus the expression $\int_{-\infty}^{\infty}|d p(\lambda)|$ need not necessarily be finite for the functions $p(\lambda)$ which we consider. We shall, however, impose a certain condition on the behaviour of $p(\lambda)$ which will enable us to form Radon-Stieltjes integrals $\left({ }^{4}\right)$ of the form $\int_{-\infty}^{\infty} h(\lambda) d p(\lambda)$ for certain classes of functions $h(\lambda)$. In fact we shall define a norm $N_{1}(p)$ for right-continuous functions $p(\lambda)$ of bounded variation on every finite interval and we shall work with functions $p(\lambda)$ of finite norm, $N_{1}(p)<\infty$. If $p(\lambda)$ and $q(\lambda)$ are two such functions, each of finite norm, then the convolution $\int_{-\infty}^{\infty} q(\lambda-\mu) d p(\mu)$ need not necessarily exist, but nevertheless a certain modified convolution can be shown to exist, namely $p \times q=\int_{-\infty}^{\infty}\left[q(\lambda-\mu)-q(-\mu) d p(\mu)=\int_{-\infty}^{\infty}[p(\lambda-\mu)-p(-\mu)] d q(\mu)\right.$. And this modified convolution serves the usual purposes required for the ordinary convolution. For example, if $P(w)$ and $Q(w)$ are the Fourier-Stieltjes transform of $p(\lambda)$ and $q(\lambda)$, respectively, $P(w)=\int_{-\infty}^{\infty} e^{\lambda w} d p(\lambda), Q(w)=\int_{-\infty}^{\infty} e^{\lambda w} d q(\lambda)$ then the product $R(w)=P(w) Q(w)$ is the Fourier-Stieltjes transform of the modified convolution $r(\lambda) \equiv p(\lambda) \times q(\lambda)$.

We will also define a (weak) norm $N(f)$ for analytic functions $f(z)$ in a strip $a<\operatorname{Im} z<b$, this norm being related to our $N_{1}$-norm, and we shall show in particular that the ordinary convolution $\int_{-\infty}^{\infty} f(z-\lambda) d p(\lambda)$ exists and yields a function analytic in $a<\operatorname{Im} z<b$ and of finite $N$-norm at most $N(f) N_{1}(p)$, whenever $N(f)$ and $N_{1}(p)$ are finite. The fact that the $N$-norm is a weak norm will be useful in our functional equations since it will enable us to treat

(4) The integrals which occur in this paper are understood to be Lebesgue-Stieltjes (Radon) integrals. See, for example, Saks, Theory of the integral, 2d revised edition, Warsaw-Lemberg, 1937, pp. 19 and 67. We note that the familiar principles of monotone and dominated convergence in the Lebesgue theory as well as the symmetric Fubini theorem are also valid in this theory; see pp. 28,29 , and 77 . In this paper we shall not admit $\pm \infty$ as members of our number system; that is, existence implies finiteness. 
analytic functions of finite exponential growth in a strip, the rate of growth being permitted to increase as the rate of growth of the function $p(\lambda)$ of bounded variation decreases.

We now proceed with the necessary definitions and lemmas. Let two positive continuous functions $E(x)$ and $E_{1}(x)$ satisfying

$$
E(x+y) \leqq E(x) E_{1}(y), \quad E_{1}(x+y) \leqq E_{1}(x) E_{1}(y),
$$

for all $x$ and $y$, be given. These functions will be designated throughout the paper by this same notation. In practice, $E(x)$ and $E_{1}(x)$ will usually be

$$
E(x)=\left\{\begin{array}{ll}
e^{\alpha x}, & x \geqq 0, \\
e^{\beta x}, & x<0,
\end{array} \quad E_{1}(x)= \begin{cases}e^{\beta x}, & x \geqq 0 \\
e^{\alpha x}, & x<0\end{cases}\right.
$$

with $\alpha<\beta$. The reader can readily verify that these functions satisfy (2.1). In terms of definite preassigned functions $E(x)$ and $E_{1}(x)$, we define the following two norms:

DEFINITION 1. Let $p(\lambda)$ be a right-continuous function of bounded variation on every finite interval. Then we define the norm

$$
N_{1}(p)=\int_{-\infty}^{\infty} E_{1}(\lambda)|d p(\lambda)|
$$

Definition 2. Let $f(z)=f(x+i y)$ be defined in a strip $a<y<b$. Then we define the norms

$$
N(f ; y)=\int_{-\infty}^{\infty} E(x)|f(x+i y)| d x
$$

and

$$
N(f)=\sup _{a<\nu<b} N(f ; y) .
$$

In practice the $N$-norm will be used for functions which are analytic in a strip. Nevertheless we use this general definition which applies to any function of a complex variable, since on occasions we will use the finiteness of the norm to aid in proving the analyticity.

We shall use the term "of finite norm" with reference to both functions of a complex variable and functions of bounded variation, with the understanding that the appropriate norm will be used in each case. While there is a possible overlapping of these types of functions, in practice no confusion will arise from the use of the term "of finite norm."

We shall use the following properties of the norm, where the existence (finiteness) of the right side implies the existence of the left. 
LEMMA 2.1.

$$
\begin{aligned}
N_{1}\left(p_{1}+p_{2}\right) & \leqq N_{1}\left(p_{1}\right)+N_{1}\left(p_{2}\right), \\
N\left(f_{1}+f_{2} ; y\right) & \leqq N\left(f_{1} ; y\right)+N\left(f_{2} ; y\right), \\
N\left(f_{1}+f_{2}\right) & \leqq N\left(f_{1}\right)+N\left(f_{2}\right), \\
N\left[\int_{-\infty}^{\infty} f(\cdot-\lambda) d p(\lambda)\right] & \leqq N_{1}(p) N(f), \\
N\left(\frac{f}{-c} ; y\right) & \leqq \frac{1}{|\operatorname{Im}(z-c)|} N(f ; y) .
\end{aligned}
$$

The inequalities (2.4), (2.5), (2.5a), (2.7) follow immediately from the definitions; so we proceed to the proof of (2.6). Obviously it is sufficient to prove

$$
N\left[\int_{-\infty}^{\infty} f(\cdot-\lambda) d p(\lambda) ; y\right] \leqq N_{1}(p) N(f ; y)
$$

and we may without loss of generality assume that $y=0$. Then we have

$$
\begin{aligned}
N\left[\int_{-\infty}^{\infty} f(\cdot-\lambda) d p(\lambda) ; 0\right] & =\int_{-\infty}^{\infty} E(x)\left|\int_{-\infty}^{\infty} f(x-\lambda) d p(\lambda)\right| d x \\
& \leqq \int_{-\infty}^{\infty} E(x) d x \int_{-\infty}^{\infty}|f(x-\lambda)||d p(\lambda)| \\
& =\int_{-\infty}^{\infty}|d p(\lambda)| \int_{-\infty}^{\infty} E(x)|f(x-\lambda)| d x \\
& \leqq \int_{-\infty}^{\infty}|d p(\lambda)| \int_{-\infty}^{\infty} E_{1}(\lambda) E(x-\lambda)|f(x-\lambda)| d x \\
& =\int_{-\infty}^{\infty} E_{1}(\lambda)|d p(\lambda)| \int_{-\infty}^{\infty} E(x)|f(x)| d x \\
& =N_{1}(p) N(f ; 0) .
\end{aligned}
$$

(It is clear from this argument that $\int_{-\infty}^{\infty}|f(x-\lambda)| \cdot|d p(\lambda)|$ and hence $\int_{-\infty}^{\infty} f(x-\lambda) d p(\lambda)$ must exist for almost all $x$ when $N_{1}(p)$ and $N(f ; 0)$ exist.)

LEMma 2.2. Let $f(z)$ be analytic in $a<\operatorname{Im} z<b$, and let $\delta>0$. Then for any point $z=x+i y$ in the strip $a+2 \delta<\operatorname{Im} z<b-2 \delta$ we have

$$
|f(z)| \leqq \frac{2}{\pi \delta} N(f) \cdot \sup _{|x-\xi|<\delta}[E(\xi)]^{-1} .
$$

This follows from the fact that $f(z)$ is a harmonic function of $x$ and $y$. Thus 
and

$$
f(z)=\frac{1}{\pi \delta^{2}} \iint_{|z-w|<\delta} f(u+i v) d u d v, \quad w=u+i v,
$$

$$
\begin{aligned}
\pi \delta^{2}|f(z)| & \leqq \int_{y-\delta}^{y+\delta} \int_{x-\delta}^{x+\delta}|f(u+i v)| d u d v \\
& \leqq \int_{y-\delta}^{y+\delta} \int_{x-\delta}^{x+\delta}|f(u+i v)|[E(u)]^{-1} E(u) d u d v \\
& \leqq \sup _{|x-\xi|<\delta}[E(\xi)]^{-1} \int_{y-\delta}^{y+\delta} \int_{-\infty}^{\infty}|f(u+i v)| E(u) d u d v \\
& \leqq \sup _{|x-\xi|<\delta}[E(\xi)]^{-1} \int_{y-\delta}^{y+\delta} N(f) d v \\
& =2 \delta \sup _{|x-\xi|<\delta}[E(\xi)]^{-1} \cdot N(f) .
\end{aligned}
$$

Lemma 2.3. If $f(z)$ is analytic in the strip $a<\operatorname{Im} z<b$ and if $N(f)$ and $N_{1}(p)$ are both finite, then $\int_{-\infty}^{\infty} f(z-\lambda) d p(\lambda)$ is analytic in the strip $a<\operatorname{Im} z<b$.

To prove this, we shall show that

$$
\int_{0}^{\infty} f(z-\lambda) d p(\lambda)=\lim _{M \rightarrow \infty} \int_{0}^{M} f(z-\lambda) d p(\lambda)
$$

uniformly in $z$ in the rectangle $R_{\delta}: a+2 \delta<\operatorname{Im} z<b-2 \delta, A+\delta<\operatorname{Re} z<B-\delta$, where $A$ and $B$ are arbitrary. Now by (2.9), (2.6), (2.2) we have in $R_{8}$ :

$$
\begin{aligned}
\left|\int_{M_{1}}^{M_{2}} f(z-\lambda) d p(\lambda)\right| & \leqq \frac{2}{\pi \delta} N\left[\int_{M_{1}}^{M_{2}} f(\cdot-\lambda) d p(\lambda)\right] \cdot \sup _{|x-\xi|<\delta}[E(\xi)]^{-1} \\
& \leqq \frac{2}{\pi \delta} N(f) \cdot\left[\int_{M_{1}}^{M_{2}} E_{1}(\lambda)|d p(\lambda)|\right] \cdot \sup _{\boldsymbol{A}<\xi<B}[E(\xi)]^{-1},
\end{aligned}
$$

since we can consider the finite integral as an infinite integral with $p(\lambda)$ replaced by a function which is constant outside the finite interval. Thus $\lim _{M_{1}, M_{2} \rightarrow \infty} \int_{M_{1}}^{M_{2}} f(z-\lambda) d p(\lambda)=0$ uniformly in $R_{\delta}$, and the uniform convergence in (2.10) is established. Since the finite integral is obviously analytic, the same is true of the infinite and also of the two-way infinite integral.

LEMMA 2.4. Let $\left\{f_{n}(z)\right\}$ be a sequence of analytic functions in the strip $a<\operatorname{Im} z<b$ such that

$$
\sum_{n=1}^{\infty} N\left(f_{n}\right)<\infty .
$$

Then the series 


$$
f(z)=\sum_{n=1}^{\infty} f_{n}(z)
$$

converges absolutely and uniformly in every closed finite region contained in the strip $a<\operatorname{Im} z<b$ and represents an analytic function $f(z)$ of finite norm in the strip. Moreover

$$
N(f) \leqq \sum_{n=1}^{\infty} N\left(f_{n}\right)
$$

and if $N_{1}(p)<\infty$, then

$$
\int_{-\infty}^{\infty}\left[\sum_{n=1}^{\infty} f_{n}(z-\lambda)\right] d p(\lambda)=\sum_{n=1}^{\infty} \int_{-\infty}^{\infty} f_{n}(z-\lambda) d p(\lambda)
$$

where the right member converges uniformly in every closed finite subregion of the strip.

The absolute and uniform convergence of (2.12) in closed finite subregions of the strip follows immediately from (2.9) and (2.11). Thus $f(z)$ is analytic in the strip and we have

$$
\begin{aligned}
N(f ; y) & =\int_{-\infty}^{\infty} E(x)|f(x+i y)| d x=\int_{-\infty}^{\infty} E(x)\left|\sum_{n=1}^{\infty} f_{n}(x+i y)\right| d x \\
& \leqq \int_{-\infty}^{\infty} E(x) \sum_{n=1}^{\infty}\left|f_{n}(x+i y)\right| d x=\sum_{n=1}^{\infty} \int_{-\infty}^{\infty} E(x)\left|f_{n}(x+i y)\right| d x \\
& =\sum_{n=1}^{\infty} N\left(f_{n} ; y\right) .
\end{aligned}
$$

Moreover if $h(z)=\int_{-\infty}^{\infty} f(z-\lambda) d p(\lambda)$ and $h_{n}(z)=\int_{-\infty}^{\infty} f_{n}(z-\lambda) d p(\lambda)$, and $a+2 \delta$ $<\operatorname{Im} z<b-2 \delta$, then

$$
\left|h(z)-\sum_{n=1}^{M-1} h_{n}(z)\right|=\left|\int_{-\infty}^{\infty} \sum_{n=M}^{\infty} f_{n}(z-\lambda) d p(\lambda)\right|
$$

[by (2.9)]

$$
\leqq \frac{2}{\pi \delta} N\left(\int_{-\infty}^{\infty} \sum_{n=M}^{\infty} f_{n}(\cdot-\lambda) d p(\lambda)\right) \sup _{|x-\xi|<\delta}[E(\xi)]^{-1}
$$

[by (2.6)] $\leqq \frac{2}{\pi \delta} N_{1}(p) N\left(\sum_{n=M}^{\infty} f_{n}\right) \sup _{|x-\xi|<\delta}[E(\xi)]^{-1}$

[by (2.13)] $\leqq \frac{2}{\pi \delta} N_{1}(p)\left[\sum_{n=M}^{\infty} N\left(f_{n}\right)\right] \sup _{|x-\xi|<\delta}[E(\xi)]^{-1}$

[by (2.11)] $\rightarrow 0$ as $M \rightarrow \infty$.

This yields (2.14). 
LEMMA 2.5. Let $p(\lambda)$ be a right-continuous function of bounded variation on every finite interval and of finite norm. For any values of $\lambda$ and $\mu$ we have

$$
\begin{aligned}
\left|p(\lambda-\mu)-p\left(-^{\prime} \mu\right)\right| & \leqq\left\{\sup _{\nu \in\left[-\mu, \lambda \_\mu\right]} \frac{1}{E_{1}(\nu)}\right\} N_{1}(p) \\
& \leqq\left\{\sup _{\nu \in[0, \lambda]} \frac{1}{E_{1}(\nu)}\right\} E_{1}(\mu) N_{1}(p)
\end{aligned}
$$

where $\left[\alpha_{1}, \alpha_{2}\right]$ denotes the closed interval with endpoints $\alpha_{1}$ and $\alpha_{2}\left(\alpha_{1} \geqq \alpha_{2}, \alpha_{1}<\alpha_{2}\right)$.

Proof.

$$
\begin{aligned}
|p(\lambda-\mu)-p(-\mu)| & =\left|\int_{-\mu}^{\lambda-\mu} d p(s)\right| \leqq\left|\int_{-\mu}^{\lambda-\mu} \frac{1}{E_{1}(s)} E_{1}(s) d p(s)\right| \\
& \leqq\left\{\sup _{\nu \in[-\mu, \lambda-\mu]} \frac{1}{E_{1}(\nu)}\right\} N_{1}(p) .
\end{aligned}
$$

The second inequality in (2.16) follows from (2.1). Thus

$$
1 / E_{1}(\nu-\mu) \leqq E_{1}(\mu) / E_{1}(\nu) .
$$

LEMMA 2.6. If $p(\lambda)$ and $q(\lambda)$ are of bounded variation on every finite interval and of finite norm then the modified convolution

$$
p \times q \equiv \int_{-\infty}^{\infty}[p(\lambda-\mu)-p(-\mu)] d q(\mu)
$$

exists and is of bounded variation on every finite interval and is of finite norm,

$$
N_{1}\left(\int_{-\infty}^{\infty}[p(\cdot-\mu)-p(-\mu)] d q(\mu)\right) \leqq N_{1}(p) N_{1}(q) .
$$

Proof. By (2.16)

$$
\begin{aligned}
& \int_{-\infty}^{\infty}|p(\lambda-\mu)-p(-\mu)||d q(\mu)| \\
& \leqq\left\{\sup _{\nu \in[0, \lambda]} \frac{1}{E_{1}(\nu)}\right\} N_{1}(p) \int_{-\infty}^{\infty} E_{1}(\mu)|d q(\mu)| \\
&=\left\{\sup _{\nu \in[0, \lambda]} \frac{1}{E_{1}(\nu)}\right\} N_{1}(p) N_{1}(q) .
\end{aligned}
$$

Thus the integral in (2.17) exists (is finite). If $p$ and $q$ are both increasing functions then the modified convolution is increasing. It readily follows that (2.17) is of bounded variation in the general case. For (2.18) we have 


$$
\begin{aligned}
N_{1}\left\{\int_{-\infty}^{\infty}[p(\lambda-\mu)\right. & -p(-\mu)] d q(\mu)\} \\
& =\int_{-\infty}^{\infty} E_{1}(\lambda)\left|d_{\lambda} \int_{-\infty}^{\infty}(p(\lambda-\mu)-p(-\mu)] d q(\mu)\right| \\
& \leqq \int_{-\infty}^{\infty} E_{1}(\lambda) d_{\lambda}\left\{\int_{-\infty}^{\infty} \operatorname{Var}[p(\lambda-\mu)-p(-\mu)]|d q(\mu)|\right\} \\
& =\int_{-\infty}^{\infty}|d q(\mu)| \int_{-\infty}^{\infty} E_{1}(\lambda)\left|d_{\lambda}[p(\lambda-\mu)-p(-\mu)]\right| \\
& =\int_{-\infty}^{\infty}|d q(\mu)| \int_{-\infty}^{\infty} E_{1}(\mu+\nu)|d p(\nu)| \\
& \leqq \int_{-\infty}^{\infty}|d q(\mu)| \int_{-\infty}^{\infty} E_{1}(\mu) E_{1}(\nu)|d p(\nu)| \\
& =N_{1}(p) N_{1}(q) ;
\end{aligned}
$$

which proves $(2.18)\left({ }^{5}\right)$.

In some places it will be useful to have our functions normalized in the following way. We call $p(\lambda)$ normalized if (i) $p(\lambda)$ is right-continuous and (ii) $p(0)=0$.

CoROllary to LEMma 2.6. If $p$ is normalized then the convolution $p \times q$ is normalized. If $p$ and $q$ are normalized then the convolution is symmetric: $p \times q=q \times p$.

Leмма 2.7. Let $\left\{p_{n}(\lambda)\right\}$ be a sequence of normalized functions, each of bounded variation on every finite interval, such that

$$
\sum_{n=1}^{\infty} N_{1}\left(p_{n}\right)<\infty
$$

Then on every finite interval the series

$$
p(\lambda)=\sum_{1}^{\infty} p_{n}(\lambda)
$$

converges uniformly to a function of bounded variation, and is a function of finite norm. Moreover

$$
N_{1}(p) \leqq \sum_{n=1}^{\infty} N_{1}\left(p_{n}\right)
$$

(5) To see that the interchange of order of integration just made is justifiable compare An unsymmetric Fubini theorem by R. H. Cameron and W. T. Martin, Bull. Amer. Math. Soc. vol. 47 (1941) pp. 121-125. Throughout the paper we shall frequently make use of the result to permit an interchange of order of integration of two integrals of this form. 
Furthermore, if $N_{1}(q)<\infty$, then

$$
\begin{aligned}
\int_{-\infty}^{\infty}\left[\sum_{n=1}^{\infty}\left\{p_{n}(\lambda-\mu)-p_{n}(-\mu)\right\}\right] d q(\mu) \\
=\sum_{n=1}^{\infty} \int_{-\infty}^{\infty}\left\{p_{n}(\lambda-\mu)-p_{n}(-\mu)\right\} d q(\mu),
\end{aligned}
$$

where in every finite interval the right member converges uniformly to a function of bounded variation.

The uniform convergence of (2.20) follows from Lemma 2.5. That the sum is of bounded variation can be seen by separating into increasing parts. Thus if

$$
\stackrel{ \pm}{p_{n}}(\lambda)=\frac{1}{2} \int_{0}^{\lambda}\left|d p_{n}(\mu)\right| \pm p_{n}(\lambda)
$$

then

and

$$
N_{1}\left(p_{n}^{ \pm}\right) \leqq N_{1}\left(p_{n}\right)
$$

$$
p(\lambda)=p^{+}(\lambda)-p^{-}(\lambda) \equiv \sum_{n=1}^{\infty} p_{n}^{+}(\lambda)-\sum_{n=1}^{\infty} p_{n}^{-}(\lambda),
$$

so that $p(\lambda)$ is of bounded variation on every finite interval.

Next, (2.21) follows for increasing parts:

$$
\begin{aligned}
& \int_{-M}^{M} E_{1}(\lambda) d {\left[p^{ \pm}(\lambda)-\sum_{n=1}^{K} p_{n}^{ \pm}(\lambda)\right] } \\
& \leqq \\
&\left.\max _{-M \leqq \lambda \leqq M} E_{1}(\lambda)\right] \int_{-M}^{M} d\left[p^{ \pm}(\lambda)-\sum_{1}^{K} p_{n}^{ \pm}(\lambda)\right] \\
&=\left[\max _{-M \leqq \lambda \leqq M} E_{1}(\lambda)\right]\left\{\left[p^{ \pm}(M)-\sum_{1}^{K} p_{n}^{ \pm}(M)\right]\right. \\
&\left.\quad-\left[p^{ \pm}(-M)-\sum_{1}^{K} p_{n}^{ \pm}(-M)\right]\right\} \\
& \rightarrow 0 \text { as } K \rightarrow \infty, \text { for fixed } M .
\end{aligned}
$$

This yields

$$
\int_{-M}^{M} E_{1}(\lambda) d p^{ \pm}(\lambda)=\sum_{n=1}^{\infty} \int_{-M}^{M} E_{1}(\lambda) d p_{n}^{ \pm}(\lambda) \leqq \sum_{n=1}^{\infty} N_{1}\left(p_{n}^{ \pm}\right) .
$$

Letting $M$ approach infinity we obtain (2.21) for increasing parts. For the general case, 
and

$$
\begin{aligned}
N_{1}(p) & =N_{1}\left(p^{+}-p^{-}\right) \leqq N_{1}\left(p^{+}\right)+N_{1}\left(p^{-}\right) \\
& \leqq \sum_{1}^{\infty} N_{1}\left(p_{n}^{+}\right)+\sum_{1}^{\infty} N_{1}\left(p_{n}^{-}\right) \leqq 2 \sum_{1}^{\infty} N_{1}\left(p_{n}\right),
\end{aligned}
$$

$$
\begin{aligned}
N_{1}\left(\sum_{n=1}^{\infty} p_{n}\right) & =N_{1}\left(\sum_{n=1}^{K} p_{n}+\sum_{n=k+1}^{\infty} p_{n}\right) \leqq N_{1}\left(\sum_{1}^{K} p_{n}\right)+N_{1}\left(\sum_{k+1}^{\infty} p_{n}\right) \\
& \leqq \sum_{1}^{K} N_{1}\left(p_{n}\right)+2 \sum_{k+1}^{\infty} N_{1}\left(p_{n}\right) \rightarrow \sum_{1}^{\infty} N_{1}\left(p_{n}\right) \text { as } K \rightarrow \infty .
\end{aligned}
$$

This yields (2.21) in general.

For (2.22) we use Lemma 2.5 and condition (2.19) and obtain

$$
\begin{aligned}
& \int_{-\infty}^{\infty} \sum_{n=1}^{\infty}\left|p_{n}(\lambda-\mu)-p_{n}(-\mu)\right||d q(\mu)| \\
& \leqq\left\{\sup _{\nu \in[0, \lambda]} \frac{1}{E_{1}(\nu)}\right\} \int_{-\infty}^{\infty} E_{1}(\mu)\left[\sum_{n=1}^{\infty} N_{1}\left(p_{n}\right)\right]|d q(\mu)| \\
&=\left\{\sup _{\nu \in[0, \lambda]} \frac{1}{E_{1}(\nu)}\right\}\left[\sum_{1}^{\infty} N_{1}\left(p_{n}\right)\right] N_{1}(q) .
\end{aligned}
$$

This enables us to apply the principle of dominated convergence to obtain (2.22). That the right member of (2.22) converges uniformly in every finite interval to a function of bounded variation follows immediately from Lemma 2.6 and the earlier part of the present lemma already proved.

3. The difference equation with constant coefficients. We shall now treat equation (1.1). We obtain a slightly more general result than Theorem 1 in terms of an integral equation.

TheOREM 1a. Let $p(\lambda)$ be a right-continuous complex-valued function of bounded variation on every finite interval, whose singular part is zero; let $p$ be of finite norm,

$$
N_{1}(p) \equiv \int_{-\infty}^{0} e^{\alpha \lambda}|d p(\lambda)|+\int_{0}^{\infty} e^{\beta \lambda}|d p(\lambda)|<\infty,
$$

and let $p$ be such that the (analytic) function

$$
P(w)=\int_{-\infty}^{\infty} e^{\lambda w} d p(\lambda)
$$

is non-vanishing in the strip $\alpha<\operatorname{Re} w<\beta$. Let $g(z)$ be a function analytic in the strip $a<\operatorname{Im} z<b$ and of finite $N$-norm,

$$
N(g) \equiv \sup _{a<y<b}\left[\int_{-\infty}^{0} e^{\beta x}|g(x+i y)| d x+\int_{0}^{\infty} e^{\alpha x}|g(x+i y)| d x\right]<\infty .
$$


Then the equation

$$
\int_{-\infty}^{\infty} f(z-\lambda) d p(\lambda)=g(z)
$$

has one and only one solution $f(z)$ which is analytic in the strip $a<\operatorname{Im} z<b$ and which is of finite norm $N(f)<\infty$.

For the proof let us notice that since $P(w)$ is analytic and non-vanishing in the strip $\alpha<\operatorname{Re} w<\beta$ and since (3.1) holds it follows from a result of Pitt (loc. cit.) that $1 / P(w)$ is expressible in the form

$$
\frac{1}{P(w)}=\int_{-\infty}^{\infty} e^{\lambda w} d p^{*}(\lambda), \quad \alpha<\operatorname{Re} w<\beta,
$$

where $p^{*}(\lambda)$ is a function of bounded variation on every finite interval with finite norm. Also( $\left.{ }^{6}\right)$ since

$$
\begin{aligned}
1 & =\frac{P(w)}{P(w)}=\int_{-\infty}^{\infty} e^{\lambda w} d p(\lambda) \int_{-\infty}^{\infty} e^{\mu v} d p^{*}(\mu) \\
& =\int_{-\infty}^{\infty} d p^{*}(\mu) \int_{-\infty}^{\infty} e^{\nu v} d \nu p(\nu-\mu) \\
& =\int_{-\infty}^{\infty} d p^{*}(\mu) \int_{-\infty}^{\infty} e^{\nu v} d_{\nu}[p(\nu-\mu)-p(-\mu)] \\
& =\int_{-\infty}^{\infty} e^{\nu v} d_{\nu} \int_{-\infty}^{\infty}[p(\nu-\mu)-p(-\mu)] d p^{*}(\mu), \quad \alpha<\operatorname{Re} w<\beta,
\end{aligned}
$$

it follows that $\int_{-\infty}^{\infty}[p(\nu-\mu)-p(-\mu)] d p^{*}(\mu)$ is a function with a unit jump at the origin and constant elsewhere. Next we form the function

$$
f(z)=\int_{-\infty}^{\infty} g(z-\mu) d p^{*}(\mu)
$$

which by Lemmas 2.3 and 2.1 is analytic in the strip $a<\operatorname{Im} z<b$, and of finite norm. Moreover

$$
\begin{aligned}
\int_{-\infty}^{\infty} f(z-\lambda) d p(\lambda) & =\int_{-\infty}^{\infty} \int_{-\infty}^{\infty} g(z-\lambda-\mu) d p^{*}(\mu) d p(\lambda) \\
& =\int_{-\infty}^{\infty} g(z-\nu) d_{\nu} \int_{-\infty}^{\infty}[p(\nu-\mu)-p(-\mu)] d p^{*}(\mu) \\
& =g(z) .
\end{aligned}
$$

Thus $f(z)$ satisfies the equation (3.4).

(6) See Footnote 4. 
For uniqueness let us assume that $\tilde{f}$ is any solution of (3.4) of finite norm. Then

$$
f(z)=\int_{-\infty}^{\infty} g(z-\mu) d p^{*}(\mu)=\int_{-\infty}^{\infty}\left[\int_{-\infty}^{\infty} \tilde{f}(z-\mu-\lambda) d p(\lambda)\right] d p^{*}(\mu)=\tilde{f}(z) ;
$$

that is, $f \equiv \tilde{f}$.

This concludes the proof of Theorem 1a, and also shows that the solution $f(z)$ is given explicitly by (3.7).

Theorem 1 is an immediate consequence of Theorem 1a in view of Lemmas 1.1 and 1.2.

4. The difference equation with one linear coefficient. We now deal with equation (1.5), and as in the previous case, we obtain a slightly more general result expressed in terms of an integral equation $\left({ }^{7}\right)$.

THEOREM 2a. Let $p(\lambda)$ be a right-continuous function of bounded variation on every finite interval and such that

$$
N_{1}(p) \equiv \int_{-\infty}^{0} e^{\alpha \lambda}|d p(\lambda)|+\int_{0}^{\infty} e^{\beta \lambda}|d p(\lambda)|<\infty .
$$

Let $y_{0}=\operatorname{Im}[p(0+0)-p(0-0)]$ and let $a<\operatorname{Im} z<b$ be any strip whose closure does not contain the line $\operatorname{Im} z=y_{0}$. Let $g(z)$ be a function analytic in the strip $a<\operatorname{Im} z<b$ and of finite norm:

$$
N(g)=\sup _{a<y<b}\left[\int_{-\infty}^{0} e^{\beta x}|g(x+i y)| d x+\int_{0}^{\infty} e^{\alpha x}|g(x+i y)| d x<\infty .\right.
$$

Then the equation

$$
z f(z)=\int_{-\infty}^{\infty} f(z-\lambda) d p(\lambda)+g(z)
$$

has one and only one solution $f(z)$ which is analytic in the strip $a<\operatorname{Im} z<b$ and which is of finite norm, $N(f)<\infty$.

We base the proof upon the following lemma.

LeMmA 4.1. Let $p(\lambda)$ be a function of bounded variation on every finite interval and of finite norm, $N_{1}(p)<\infty$. Let $z_{1}=x_{1}+i y_{1}$ be any complex number, and let $a<\operatorname{Im} z<b$ be any strip whose distance from the line $\operatorname{Im} z=y_{1}$ is greater than $N_{1}(p)$. Let $g(z)$ be a function analytic in the strip $a<\operatorname{Im} z<b$ for which $N(g)<\infty$. Then the equation

$\left({ }^{7}\right)$ This result and its proof were suggested by applying certain duality considerations to a corresponding result on differential equations. See R. H. Cameron, Quadratures involving trigonometric sums, Journal of Mathematics and Physics vol. 19 (1940) pp. 161-166. This result is however certainly not a direct dual since $g(z)$ need not have any Fourier transform. 


$$
\left(z-z_{1}\right) f(z)=\int_{-\infty}^{\infty} f(z-\lambda) d p(\lambda)+g(z)
$$

has one and only one solution $f(z)$ of finite norm and analytic in the strip $a<\operatorname{Im} z<b$. In addition

$$
N[f ; y] \leqq \frac{N(g ; y)}{\left|y-y_{1}\right|-N_{1}(p)}, \quad a<y<b .
$$
Let

and

$$
f_{0}(z)=g(z) /\left(z-z_{1}\right)
$$

$$
f_{n}(z)=\frac{1}{z-z_{1}} \int_{-\infty}^{\infty} f_{n-1}(z-\lambda) d p(\lambda), \quad n=1,2, \cdots,
$$

each $f_{n}(z)$ existing in view of Lemma 2.1. We shall show that

$$
f(z)=\sum_{n=0}^{\infty} f_{n}(z)
$$

also exists and satisfies the conclusions of Lemma 4.1. First, by Lemma 2.1,

and

$$
N\left(f_{0} ; y\right) \leqq \frac{1}{\left|y-y_{1}\right|} N(g ; y)
$$

$$
\begin{aligned}
N\left(f_{n} ; y\right) \leqq \frac{1}{\left|y-y_{1}\right|} N\left(f_{n-1} ; y\right) N_{1}(p) \leqq & \frac{\left[N_{1}(p)\right]^{n}}{\left|y-y_{1}\right|^{n+1}} N(g ; y), \\
& a<g<b, n=1,2, \cdots .
\end{aligned}
$$

Thus

$$
\sum_{n=0}^{\infty} N\left(f_{n} ; y\right) \leqq \sum_{n=0}^{\infty} \frac{\left[N_{1}(p)\right]^{n}}{\left|y-y_{1}\right|^{n+1}} N_{1}(g ; y)=\frac{N(g ; y)}{\left|y-y_{1}\right|-N_{1}(p)} .
$$

By Lemmas 2.4, 2.2, and equation (4.8) we see that the function defined by (4.7) is analytic and of finite norm in the strip $a<\operatorname{Im} z<b$ and that (4.5) holds. that

To see that the $f$ defined by (4.7) satisfies equation (4.4) we note by (4.6)

(4.9) $f(z)=f_{0}(z)+\sum_{n=1}^{\infty} f_{n}(z)=-\frac{1}{z-z_{1}}\left[g(z)+\sum_{n=1}^{\infty} \int_{-\infty}^{\infty} f_{n-1}(z-\lambda) d p(\lambda)\right]$.

By Lemma 2.4, we can interchange the order of summation and integration and thus 


$$
f(z)=\frac{1}{z-z_{1}}\left[g(z)+\int_{-\infty}^{\infty} f(z-\lambda) d p(\lambda)\right]
$$

that is, $f(z)$ satisfies equation (4.4).

For uniqueness let us assume that $f^{*}(z)$ is a solution of (4.4), analytic in $a<\operatorname{Im} z<b$ and of finite norm. Then

$$
f^{*}(z)-f(z)=\frac{1}{z-z_{1}} \int_{-\infty}^{\infty}\left[f^{*}(z-\lambda)-f(z-\lambda)\right] d p(\lambda)
$$

and

or

$$
N\left(f^{*}-f ; y\right) \leqq \frac{1}{\left|y-y_{1}\right|} N\left(f^{*}-f ; y\right) N_{1}(p), \ldots a<y<b,
$$

$$
N\left(f^{*}-f ; y\right)\left(1-\frac{N_{1}(p)}{\left|y-y_{1}\right|}\right) \leqq 0, \quad a<y<b .
$$

Since the second factor is positive, $f^{*}-f \equiv 0$.

This concludes the proof of Lemma 4.1.

We now proceed to the proof of Theorem 2a itself. Let us therefore return to the notation of that theorem and assume that the hypotheses of the theorem are satisfied. We begin by introducing a transformation which transforms equation (4.3) into one to which the preceding lemma applies. This transformation will be based upon the following function:

$$
R(w)=\exp \left[\int_{-\infty}^{-\eta} \frac{e^{\lambda w}}{\lambda} d p(\lambda)+\int_{\eta}^{\infty} \frac{e^{\lambda w}}{\lambda} d p(\lambda)\right], \quad \alpha<\operatorname{Re} w<\beta,
$$

the $\eta$ being chosen positive and so small that

$$
N_{1}\left(p_{1}\right)<\min \left\{\left|y_{0}-a\right|,\left|y_{0}-b\right|\right\},
$$

where

$$
p_{1}(\lambda)=\left\{\begin{array}{lr}
p(\eta)-p(0+0), & \lambda \geqq \eta, \\
p(\lambda)-p(0+0), & 0 \leqq \lambda \leqq \eta, \\
p(\lambda)-p(0-0), & -\eta \leqq \lambda<0, \\
p(-\eta)-p(0-0), & \lambda \leqq-\eta .
\end{array}\right.
$$

This is possible since by hypothesis $y_{0}$ lies outside the closed interval $a \leqq y \leqq b$ so the right member of (4.12) is positive, and the variation of $p_{1}(\lambda)$ can be made arbitrarily small due to the continuity of $p_{1}(\lambda)$ at the origin. Then if

$$
j(\lambda)=\left\{\begin{aligned}
0, & \lambda \geqq 0, \\
-1, & \lambda<0
\end{aligned}\right.
$$


and $\left(^{8}\right) z_{0}=x_{0}+i y_{0}=p(0+0)-p(0-0)$, we have

$$
R(w)=\exp \left\{\int_{-\infty}^{\infty} \frac{e^{\lambda w}}{\lambda} d\left[p(\lambda)-p_{1}(\lambda)-z_{0} j(\lambda)\right]\right\} .
$$

We next show that there exist functions $r(\lambda)$ and $r^{*}(\lambda)$ such that

$$
R(w)=\int_{-\infty}^{\infty} e^{\lambda w} d r(\lambda)
$$

and

$$
\frac{1}{R(w)}=\int_{-\infty}^{\infty} e^{\lambda w} d r^{*}(\lambda)
$$

and

$$
j(\lambda)=\int_{-\infty}^{\infty}[r(\lambda-t)-r(-t)] d r^{*}(t) .
$$

For let $q_{0}(\lambda)=j(\lambda)$ and

$$
q_{1}(\lambda)=\int_{0}^{\lambda+0} \frac{d\left[p(\xi)-p_{1}(\xi)-z_{0} j(\xi)\right]}{\xi}
$$

and

$$
q_{n}(\lambda)=\int_{-\infty}^{\infty}\left[q_{1}(\lambda-\mu)-q_{1}(-\mu)\right] d q_{n-1}(\mu), \quad n=2,3, \cdots .
$$

Since $q_{0}$ and $q_{1}$ are normalized it follows by the corollary to Lemma 2.6 that all the $q_{n}$ are normalized.

Now

$$
\int_{-\infty}^{\infty} e^{\lambda w} d q_{n}(\lambda)=\left[\int_{-\infty}^{\infty} e^{\lambda w} d q_{1}(\lambda)\right]^{n}, \quad n=0,1,2, \cdots
$$

Next define

$$
r(\lambda)=\sum_{n=0}^{\infty} \frac{1}{n !} q_{n}(\lambda)
$$

and

$$
r^{*}(\lambda)=\sum_{n=0}^{\infty} \frac{(-1)^{n}}{n !} q_{n}(\lambda)
$$
rem $2 \mathrm{a}$.

(8) Note that this is consistent with the definition of $y_{0}$ given in the statement of Theo- 
By inspection $q_{0}(\lambda)$ is of finite norm, and the same is true of $q_{1}(\lambda)$, when we note that the function with respect to which we integrate is constant in a neighborhood of the origin. By equation (2.18) it follows that each $q_{n}$ is of finite norm with $N_{1}\left(q_{n}\right) \leqq\left[N_{1}\left(q_{1}\right)\right]^{n}$, and by Lemma 2.7 that $r(\lambda)$ and $r^{*}(\lambda)$ defined in (4.20) and (4.21) exist and are of finite norm. Then

$$
\begin{aligned}
R(w) & =\exp \left\{\int_{-\infty}^{\infty} \frac{e^{\lambda w}}{\lambda} d\left[p(\lambda)-p_{1}(\lambda)-z_{0} j(\lambda)\right]\right\} \\
& =\exp \left\{\int_{-\infty}^{\infty} e^{\lambda w} d q_{1}(\lambda)\right\} \\
& =\sum_{n=0}^{\infty} \frac{1}{n !}\left[\int_{-\infty}^{\infty} e^{\lambda w} d q_{1}(\lambda)\right]^{n}=\sum_{n=0}^{\infty} \frac{1}{n !} \int_{-\infty}^{\infty} e^{\lambda w} d q_{n}(\lambda) .
\end{aligned}
$$

We shall show that the integration and summation can be interchanged, thus giving

$$
R(w)=\int_{-\infty}^{\infty} e^{\lambda w} d\left[\sum_{n=0}^{\infty} \frac{1}{n !} q_{n}(\lambda)\right]=\int_{-\infty}^{\infty} e^{\lambda w} d r(\lambda)
$$

The interchange is justified by the following argument:

$$
\begin{aligned}
\sum_{n=0}^{\infty} \frac{1}{n !} \int_{-M}^{M} & e^{\lambda w} d q_{n}(\lambda) \\
& =\sum_{n=0}^{\infty} \frac{1}{n !}\left[e^{w M} q_{n}(M)-e^{-w M} q_{n}(-M)-w \int_{-M}^{M} q_{n}(\lambda) e^{\lambda w} d \lambda\right] \\
& =e^{w M} r(M)-e^{-w M} r(-M)-w \int_{-M}^{M} r(\lambda) e^{\lambda w} d \lambda=\int_{-M}^{M} e^{\lambda w} d r(\lambda) .
\end{aligned}
$$

By the Weierstrass $M$-test and the convergence of

$$
\sum_{n=1}^{\infty} \frac{1}{n !} N_{1}\left(q_{n}\right)
$$

it follows that the above series in the first member converges uniformly for all real $M$ and thus as $M \rightarrow \infty$ the limit of the sum is the sum of the limits. Thus (4.15) holds; and it is obvious that (4.16) can be proved in the same way. Moreover, (4.15) and (4.16) imply (4.17). Returning now to (4.14), we differentiate and obtain

$$
d R(w) / d w=R(w)\left[P(w)-P_{1}(w)-z_{0}\right]
$$

where

$$
P(w)=\int_{-\infty}^{\infty} e^{\lambda w} d p(\lambda), \quad P_{1}(w)=\int_{-\infty}^{\infty} e^{\lambda w} d p_{1}(\lambda)
$$


Rewriting (4.22) by replacing each side by the function of which it is a Fourier-Stieltjes transform, and noting that

$$
\frac{d R(w)}{d w}=\int_{-\infty}^{\infty} e^{\lambda w} d \lambda \int_{0}^{\lambda} \mu d r(\mu)
$$

we obtain

$$
\int_{0}^{\lambda} \mu d r(\mu)=\int_{-\infty}^{\infty}[r(\lambda-\mu)-r(-\mu)] d\left[p(\mu)-p_{1}(\mu)-z_{0} j(\mu)\right] .
$$

Now consider the functional equation

$$
\left(z-z_{0}\right) f_{1}(z)=\int_{-\infty}^{\infty} f_{1}(z-\lambda) d p_{1}(\lambda)+g_{1}(z)
$$

where

$$
g_{1}(z)=\int_{-\infty}^{\infty} g(z-\lambda) d r^{*}(\lambda)
$$

It is clear by (4.12) that the functional equation (4.24) satisfies the hypothesis of Lemma 4.1, and thus has one and only one analytic solution $f_{1}(z)$ of finite norm in $a<\operatorname{Im~} z<b$. We can therefore consider that $f_{1}(z)$ is completely defined by (4.24), and in terms of it we define

$$
f(z)=\int_{-\infty}^{\infty} f_{1}(z-t) d r(t), \quad a<\operatorname{Im} z<b .
$$

We shall show that this $f(z)$ is the unique analytic solution of finite norm of the original equation (4.3). By Lemmas 2.3 and 2.1, $f(z)$ so defined is analytic and of finite norm. Also by (4.24) we have

$$
\begin{aligned}
\int_{-\infty}^{\infty}\left(z-t-z_{0}\right) f_{1}(z-t) d r(t) & \\
= & \int_{-\infty}^{\infty} d r(t) \int_{-\infty}^{\infty} f_{1}(z-\lambda-t) d p_{1}(\lambda)+\int_{-\infty}^{\infty} g_{1}(z-t) d r(t) .
\end{aligned}
$$

Transposing, simplifying, and using (4.25) and (4.17), we find

$$
\begin{aligned}
z \int_{-\infty}^{\infty} f_{1}(z-t) d r(t)= & \int_{-\infty}^{\infty} f_{1}(z-t) t d r(t)+z_{0} \int_{-\infty}^{\infty} f_{1}(z-t) d r(t) \\
& +\int_{-\infty}^{\infty} f_{1}(z-t) d_{t} \int_{-\infty}^{\infty}[r(t-\lambda)-r(-\lambda)] d p_{1}(\lambda)+g(z) .
\end{aligned}
$$

By (4.26) and (4.23), this yields 


$$
\begin{aligned}
z f(z) & =\int_{-\infty}^{\infty} f_{1}(z-t) d_{\imath} \int_{-\infty}^{\infty}[r(t-\lambda)-r(-\lambda)] d\left[p(\lambda)-z_{0} j(\lambda)\right]+z_{0} f(z)+g(z) \\
& =\int_{-\infty}^{\infty}\left[\int_{-\infty}^{\infty} f_{1}(z-\mu-\lambda) d r(\mu)\right] d p(\lambda)+g(z) \\
& =\int_{-\infty}^{\infty} f(z-\lambda) d p(\lambda)+g(z) .
\end{aligned}
$$

Thus $f(z)$, defined in (4.26), satisfies the original equation (4.3).

Similarly if any $f(z)$ satisfies (4.3), $f(z)$ assumed analytic and of finite norm in $a<\operatorname{Im} z<b$, it is seen that the function

$$
f_{1}(z)=\int_{-\infty}^{\infty} f(z-\lambda) d r^{*}(\lambda)
$$

satisfies equation (4.24). Thus the uniqueness of the solution of (4.24) implies the uniqueness of the solution of (4.3).

This concludes the proof of Theorem $2 \mathrm{a}$.

Theorem 2 follows from Theorem $2 \mathrm{a}$ and Lemmas 1.1 and 1.2.

5. The difference equation with linear coefficients.

THEOREM 3a. Let $p(\lambda)$ and $q(\lambda)$ be complex-valued functions of bounded variation on every finite interval such that

$$
N_{1}(p)<\infty, N_{1}(q)<\infty \text {, singular part of } p=0 .
$$

Moreover let the analytic function

$$
P(w)=\int_{-\infty}^{\infty} e^{\lambda w} d p(\lambda)
$$

be non-vanishing in the strip $\alpha<\operatorname{Re} w<\beta$. Define $\left({ }^{9}\right)$

$$
y_{0}=\operatorname{Im}\left[\lim _{T \rightarrow \infty} \frac{1}{T i} \int_{\gamma}^{\gamma+T i} \frac{\int_{-\infty}^{\infty} e^{\lambda w} d q(\lambda)}{\int_{-\infty}^{\infty} e^{\lambda w} d p(\lambda)} d w\right] .
$$

Let $a<\operatorname{Im} z<b$ be any strip whose closure does not contain the line $\operatorname{Im} z=y_{0}$. Let $g(z)$ be a function analytic in the strip $a<\operatorname{Im} z<b$ and of finite norm,

$$
N(g)<\infty \text {. }
$$

Then the equation

$$
\int_{-\infty}^{\infty}(z-\lambda) f(z-\lambda) d p(\lambda)-\int_{-\infty}^{\infty} f(z-\lambda) d q(\lambda)=g(z)
$$

has one and only one analytic solution $f(z)$ of finite norm in the strip $a<\operatorname{Im} z<b$.

(9) This limit exists and is independent of $\gamma$ for $\alpha<\gamma<\beta$. 
For the proof we again use Pitt's theorem (loc. cit.) which yields the result that $1 / P(w)$ is expressible in the form (3.5) with $N_{1}\left(p^{*}\right)<\infty$.

We next convolve equation (5.5) with $p^{*}(\mu)$ and obtain

$$
\begin{aligned}
\int_{-\infty}^{\infty} \int_{-\infty}^{\infty}(z-\lambda-\mu) f(z-\lambda-\mu) d p(\lambda) d p^{*}(\mu) \\
\quad+\int_{-\infty}^{\infty} \int_{-\infty}^{\infty} f(z-\lambda-\mu) d q(\lambda) d p^{*}(\mu)=\int_{-\infty}^{\infty} g(z-\mu) d p^{*}(\mu),
\end{aligned}
$$

or

$$
z f(z)-\int_{-\infty}^{\infty} f(z-\lambda) d \tilde{q}(\lambda)=\tilde{g}(z)
$$

where

$$
\tilde{q}(\lambda)=\int_{-\infty}^{\infty}[q(\lambda-\mu)-q(-\mu)] d p^{*}(\mu), \quad \tilde{g}(z)=\int_{-\infty}^{\infty} g(z-\mu) d p^{*}(\mu) .
$$

We note by Lemmas $2.3,2.6$, and 2.1 that $\tilde{g}(z)$ is analytic in $a<\operatorname{Im} z<b$ and that

$$
N_{1}(\tilde{q})<\infty, \quad N(\tilde{g})<\infty
$$

Moreover $\left({ }^{10}\right)$

$$
y_{0}=\operatorname{Im}[\tilde{q}(0+0)-\tilde{q}(0-0)] .
$$

Now we apply Theorem $2 \mathrm{a}$ to (5.6). We note that the hypotheses of that theorem are satisfied with $p$ and $g$ replaced by $\tilde{q}, \tilde{g}$. Thus there is a unique analytic solution $f(z)$ of (5.6) of finite norm. Hence if (5.5) has an analytic solution of finite norm it is unique. But the solution of (5.6) obviously satisfies (5.5) as we see by convolving equation (5.6) with $p$. This yields the theorem.

As in the earlier cases it is easily seen that Theorem 3 follows from Theorem 3a.

(10) That (5.9) agrees with (5.3) is easily seen.

Massachusetts Institute of Technology,

Cambridge, Mass. 